\title{
The X-ray View of Abell 3120
}

\author{
Mark Henriksen \& Alexis Finoguenov \\ Physics Department, University of Maryland, Baltimore County, Baltimore, MD 21250
}

Received _ ; accepted _

To Appear in the Astrophysical Journal Letters 


\begin{abstract}
Identification of Abell 3120 as a galaxy cluster has recently been questioned with alternative suggestions including: a fossil remnant of a group merger, nonthermal emission from a radio galaxy, and projected emission from of a filamentary string of galaxies. We report on our analysis of the Chandra observation and evaluate these hypotheses based on our results. Abell 3120 shows X-ray emission extending $158 \mathrm{kpc}$, well beyond the central galaxy. The spatial distribution of X-rays in the core more closely follows the radio emission showing a jet-like structure extending to the north that is misaligned with the stellar light distribution of the central galaxy. At larger radii the X-ray emission is aligned with the SE-NW running axis of the galaxy distribution in the cluster core. Modeling the X-ray spectrum excludes purely non-thermal emission. The emission weighted temperature is $1.93-2.19 \mathrm{keV}$ and the $0.3-10 \mathrm{keV}$ luminosity is $1.23 \times 10^{43}$ ergs $\mathrm{s}^{-1}$. Abell 3120 appears to be a poor cluster with Virgo and MKW 4 as peers. The best fitting model consists of a thermal component and a second component that may be either thermal or non-thermal, with luminosity $\sim 25 \%$ of the total X-ray luminosity. While, a more detailed spatial-spectral search failed to detect a central AGN, there is some evidence for an extended hard X-ray component. Cooler gas, 1.28 - 1.80 was detected in the central $20 \mathrm{kpc}$. The second thermal component marginally requires a higher redshift, $>0.12$, which may be due to a second cluster in the rich surrounding environment consisting of nearly a thousand catalogued galaxies.
\end{abstract}

Subject headings: X-ray: Galaxy Clusters: Abell 3120 


\section{Introduction}

Abell 3120 is located near the center of the Horologium-Reticulum Supercluster. An extended X-ray source was found with ROSAT and identified with the 2MASX J03215645-5119357 galaxy. The emission was characterized as the fossil remnant of galaxy group merger (Romer et al. 2000). Sparse kinematical data shows a high velocity dispersion around the central galaxy and it was suggested, alternatively, that the X-ray emission may due to a filament or other line of sight structure (Fleenor et al. 2006). Since there is a radio source identified with the central early-type galaxy, these authors also suggested that the X-ray emission may be wholly non-thermal and identified with the radio galaxy. A detailed study of the X-ray emission with high spatial and spectral resolution can play an important role in evaluating these three hypotheses and we report here on the X-ray characteristics of Abell 3120 using Chandra observations.

\section{Optical Analysis}

Abell 3120 is classified as a poor cluster (Abell, Corwin, \& Olowin, 1989). However, the region is actually quite rich with 813 galaxies catalogued by the APM survey (Maddox et. al., 1990) within $3 \mathrm{Mpc}$ of the cluster center. The DSS red band image has been smoothed with a $40 \mathrm{kpc}$ Gaussian (at the redshift of Abell 3120) to bring out regions of high surface brightness, including bright galaxies and areas of high galaxy density. Figure 1 shows a contour map overlaid on the smoothed DSS - R band image, $650 \mathrm{kpc}$ on a side. Substructure is visible in the optical image. Within $13.2 \operatorname{arcmin}$, or $1 \mathrm{Mpc}\left(\mathrm{H}_{o}=73 \mathrm{~km}\right.$ $\mathrm{sec}^{-1} \mathrm{Mpc}^{-1}, \Omega_{m}=0.27, \Omega_{v}=0.73$ and using the central radio galaxy's redshift of 0.0699 corrected to the reference frame defined by the Cosmic Microwave Background Radiation, the scale is $1.284 \mathrm{kpc}_{\operatorname{arcsec}^{-1}}$ ) in projection from the Abell 3120 center, there are only 7 galaxies with measured redshift (see Table 1). The mean velocity for these galaxies is 
$21236 \mathrm{~km} \mathrm{~s}^{-1}$ with a dispersion of $2824 \mathrm{~km} \mathrm{~s}^{-1}$. The galaxy with the highest velocity is more than $2 \sigma$ from the mean. If it is removed, then the mean is lowered to $20248 \mathrm{~km} \mathrm{~s}^{-1}$ and the dispersion is significantly lowered to $1416 \mathrm{~km} \mathrm{~s}^{-1}$. However, this is still a very high dispersion, even for a very high mass cluster, if it is in virial equilibrium. Figure 2 shows the optical contours overlaid on the X-ray image. The radio galaxy associated with the central galaxy is centered on the X-ray peak. A potential problem with this "clipped-mean" is that it gives a very high peculiar velocity for the central galaxy, $792 \mathrm{~km} \mathrm{~s}^{-1}$. This is a significantly higher peculiar velocity than is typical for central galaxies associated with clusters showing substructure (Oegerle \& Hill, 2001). If we assume that the radio galaxy is at rest in the cluster potential and recalculate the mean velocity relative to it, the dispersion is even higher, $1,617 \mathrm{~km} \mathrm{~s}^{-1}$. One must conclude that if these galaxies are representative of the cluster as a whole, it is unlikely that even the bulk flow from merging substructures could account for such a large velocity dispersion, given the relatively low mass cluster implied by the X-ray temperature (Section 3.3). An alternative hypothesis is that there is more than more cluster in the region sampled by the redshifts.

\section{X-ray Observations and Analysis}

Abell 3120 was observed on June 3, 2006 for approximately 27,130 seconds with the ACIS-I detector. The event 2 file produced from reprocessing III was used for both the spectral and spatial analysis. Processing includes corrections due to time dependent gain, charge transfer inefficiency (CTI), dead area correction, and bad pixels. The full energy band was used to extract the spectrum, however, during spectral fitting the energy band was limited to $0.3-10 \mathrm{keV}$. The mkacisrmf tool was used to create the response matrix file (rmf). A circular region, centered on the visual X-ray peak with radius 250 pixels or 123 arcsec, using the ACIS scale of $0.492 \operatorname{arcsec}$ pixel $^{-1}$, was used to extract the source 
spectrum. This region includes all of the X-ray emission visible in the radial profile (Figure 3). Three point sources within the source region were excluded. Three background regions were chosen in source free regions of the same chip as the source to extract a background spectrum. The analyzed spectrum for the Abell 3120 cluster has an exposure time of 26,784 seconds and a background subtracted count rate of $0.1645+/-0.0032$ counts per second. Both simple and complex models were fit to the spectrum including: (1) thermal, (2) powerlaw, (3) thermal plus powerlaw, (4) two thermal, (5) thermal with two redshifts, and (6) two thermal with different redshifts. In addition, a spatial analysis was done consisting of visually correlating emission in several energy bands, X-ray, K, R, and radio, as well as fitting radial X-ray surface brightness profiles.

\subsection{Spatial Analysis}

We fit the standard profile, $\mathrm{S} / \mathrm{S}_{c}=\left(1+\left(\mathrm{r} / \mathrm{r}_{c}\right)^{2}\right)^{-3 \beta+0.5}$ to the radial surface brightness distribution in 3 energy bands: soft $(0.3-2 \mathrm{keV})$, hard $(2-10 \mathrm{keV})$, and total. The radial profile for the 0.3 - $10 \mathrm{keV}$ energy band is shown in Figure 3. The best fit values are $\mathrm{r}_{c}=$ $7.6 \mathrm{kpc}$ and $\beta=0.441$ with $90 \%$ confidence intervals of $6.9-8.4 \mathrm{kpc}$ and $0.434-0.448$, respectively. These are comparable to $\mathrm{MKW} 4$, which is characterized by $\mathrm{r}_{c}=4.28-4.59$ $\mathrm{kpc}$ and $\beta=0.446-0.448$. The value of $\mathrm{r}_{c}$ and $\beta$ differ significantly from rich clusters, which have larger beta and larger core radii (Vikhlinin, Forman \& Jones 1999), and is typical of groups of galaxies (Mulchaey et al. 2003). The radial surface brightness profile shows emission out to 250 pix or 123 arc sec $(158 \mathrm{kpc})$, well beyond the central galaxy and out into the cluster potential. Within this region there are 16 catalogued galaxies. Thus, while the X-ray gas may be, in large part, trapped by the galaxy gravitational potential in the central region, it smoothly connects to the intracluster medium. Because there is a radio source associated with the cluster, we fit radial profiles in a soft, $0.3-2 \mathrm{keV}$ band, 
and a hard, 2 - $10 \mathrm{keV}$ band, to search for non-thermal emission in the X-ray. The $90 \%$ confidence intervals are $\mathrm{r}_{c}=6.1-7.6 \mathrm{kpc}$ and $\beta=0.433-0.451$ for the soft band and $\mathrm{r}_{c}=$ $6.6-14.1 \mathrm{kpc}$ and $\beta=0.374-0.439$ for the hard band. The central count rate is 8.8 times higher in the soft band compared to the hard band. The predominance of soft emission in the center is reflected in the fit parameter $\beta$, which is nearly identical to the total band. The beta model is flat in the center and fits both profiles well. It appears that there is no hard, central point source that can be identified with an AGN. However, the hard band has a larger core radius and a smaller beta making it significantly flatter than the soft emission. This can be seen clearly in the radial profiles (Figures 4 and 5).

\subsection{X-ray Spectral Results}

\subsection{Single Component Models}

While a single thermal component is not the best fitting model for the ACIS spectrum, the results (Table 2) are useful for comparison to correlations found in surveys of X-ray emission from galaxies, groups, and clusters. The X-ray luminosities and temperatures shown in the correlations with velocity dispersion for groups and clusters (Jeltema et al. 2006; Ortiz-Gil et al. 2004; Zimer, Zabludoff, \& Mulchaey 2003) range from $5 \times 10^{44}-10^{46}$ for clusters with a velocity dispersion above $1000 \mathrm{~km} \mathrm{~s}^{-1}$. This is a factor of almost $50-1000$ above the X-ray luminosity for Abell 3120, $1.23 \times 10^{43} \mathrm{ergs} \mathrm{s}^{-1}$ (Table 3). The temperature of Abell 3120, $1.93-2.19 \mathrm{keV}$, is also lower than predicted by the cluster correlations for a high velocity dispersion (Jeltema et al. 2006; Ortiz-Gil et al. 2004). Clusters with that temperature range in the correlations have velocity dispersions in the range, 350 - $600 \mathrm{~km}$ $\mathrm{s}^{-1}$. Though only weakly constrained by the X-ray data, the X-ray determined redshift range, $\mathrm{z}=0.069\left(20,700 \mathrm{~km} \mathrm{~s}^{-1}\right)-0.087\left(26,100 \mathrm{~km} \mathrm{~s}^{-1}\right)$, while in agreement with the redshift of the radio galaxy, gives a lower bound that excludes the dynamical model based 
on the galaxies, as discussed in section 2 , with $90 \%$ confidence. Thus we conclude that the velocity dispersion is unrelated to the Abell 3120 cluster based on cluster correlations.

Comparison to galaxy group properties show that it is somewhat unlikely that the $\mathrm{X}$-ray emission is from a galaxy group or single galaxy (fossil remnant of a group merger). The emission weighted temperature, $1.93-2.19 \mathrm{keV}$ and luminosity, $1.23 \times 10^{43} \mathrm{ergs} \mathrm{s}^{-1}$ are both high compared to groups (Ponman et al. 1996; Jeltema et al. 2006). Only HCG 62 and the NGC 5044 group have a comparable luminosity to Abell 3120. However, their temperature is significantly lower. The distribution of temperature for galaxy clusters shows very few that are around $2 \mathrm{keV}$ (Mulchaey et al. 2003; Heldson \& Ponman 2000)

The correlation between cluster X-ray luminosity and temperature spanning groups, poor clusters, and rich clusters (Ortiz-Gil et al. 2004; Jeltema et al. 2006), shows that Abell 3120 is typical of low X-ray luminosity clusters. The peer class of objects for Abell 3120 appears to be poor clusters with dominant central galaxies. Two specific examples, both comparable in their X-ray properties, are MKW 4 and Virgo. MKW 4, centered on NGC 4073, is a poor cluster which has a temperature of $1.75-1.81 \mathrm{keV}$ in the central 2 arc min (O'sullivan et al. 2003). MKW 4 has a temperature inversion in the center and reaches an ambient cluster temperature of $\sim 3 \mathrm{keV}$ so that the emission weighted temperature of the ambient intracluster medium may be somewhat higher. The MOS spectrum provides an emission weighted temperature of $2.5-3.82 \mathrm{keV}$ for this cluster. Abell 3120 has an average abundance, $0.45-0.82$ Solar, which is higher than is typical for rich clusters, 0.3 Solar, yet also comparable to MKW 4, 0.76 - 0.87 (Fukazawa et al. 2004). The spectrum of both MKW 4 and Abell 3120 is better fit by a two component model with the additional component being either thermal or powerlaw.

The Rossi X-ray Timing Explorer observation of Virgo gives a temperature of 2.56 +/$0.03 \mathrm{keV}$ and an abundance of $0.26+/-0.02$ Solar (Reynolds, et al. 1999). The emission 
weighted temperature breaks down into a two phase distribution in the inner 10 arcmin with one component being isothermal around $1 \mathrm{keV}$ and the second increasing from 1.74 $\mathrm{keV}$ in the center to $2.5 \mathrm{keV}$ at around 12 arcmin (Matsushita et al. 2002). The abundance

of alpha elements shows a decrease from approximately solar in the center to approximately 0.3 solar at 12 arcmin. Because of the large field of view of the RXTE PCA, its values are more weighted toward the cluster emission than the cooler, high abundance gas associated with M87, which may account for the lower abundance.

\subsection{Multiple Thermal Components}

The single thermal component model has a reduced chi-square of 1.17 . The probability of exceeding that value is $5 \%$, under the assumption that it is a good description of the data. Based on this low probability, we fit more complex models to the data and obtain the following results via the F-test. The $\mathrm{F}$ value is $13.2,16.3$, and 15.5 for adding either a powerlaw, second thermal, or second redshift, respectively. This means that all additional parameters (see Table 2) have greater than 99.9\% significance. Allowing the second thermal component to have an independent redshift has an $\mathrm{F}$ parameter of 2.7 and a significance of $90 \%$.

For the two thermal component model, temperatures and luminosities are $1.06-1.37$ $\mathrm{keV}\left(8.9 \times 10^{42} \mathrm{ergs} \mathrm{s}^{-1}\right)$ and $2.41-4.31 \mathrm{keV}\left(3.9 \times 10^{42} \operatorname{ergs~s}^{-1}\right)$. The low temperature component and luminosity is consistent with the L-T correlation while the high temperature component is quite underluminous. Alternatively, the high temperature component may be significantly hotter than that predicted by the correlation. Because the hot component is relatively small, $\sim 30 \%$ of the total luminosity, it may be a clump of non-virialized, shocked gas from a small mass merger. On the scale of $\sim 100 \mathrm{kpc}$, the X-ray emission appears asymmetrical and elongated along the NW-SE axis. This alignment is similar to the optical 
light distribution (Figure 2), which traces the galaxy distribution on a much larger scale. The non-spherical distribution is typical of a non-virialized cluster that has experienced recent merger and accretion events.

A comparison of the our two component model parameters for Abell 3120 to those obtained for early-type galaxies indicates that it is unlikely that the emission is due to a single galaxy. The sample of early-type galaxies observed with the Advanced Satellite for Cosmology and Astrophysics (ASCA) shows that the two component galaxy model consists

of a soft one, with $0.3 \mathrm{keV}<\mathrm{kT}<1.0 \mathrm{keV}$ and luminosity between $6.6 \times 10^{39}-2.6 \times 10^{42}$ and a harder one (Matsumota et al. 1997) . The hard component can be fit by a $\sim 10 \mathrm{keV}$ thermal or $\alpha \sim 1.8$ powerlaw with luminosity between $3.0 \times 10^{40}-3.1 \times 10^{41}$. Comparison with Abell 3120 shows that for either two component model: two thermal components or thermal plus powerlaw (next section), the hard component in Abell 3120 exceeds the maximum predicted galactic hard component luminosity by an order of magnitude. Thus, a wholly galactic origin is unlikely. The interpretation of the hard component as shocked gas in the two thermal component model is also somewhat problematic since the amount of high temperature gas is quite large when compared to simulations of relatively small merger events.

\subsection{Non-thermal Emission}

A single non-thermal component is a poor fit to the data giving a reduced $\chi^{2}$ of 2.5 . Thus, one can conclude that some of the emission must be thermal. A combination of thermal and power-law emission provides a better fit than a single thermal and it is an equally good fit to the model with two thermal components. In this model, the temperature is reduced to $1.30-1.78 \mathrm{keV}$ and the luminosity of the thermal component is reduced to $10^{43} \operatorname{ergs~s}^{-1}$. 
The powerlaw luminosity is $4.2 \times 10^{42} \mathrm{ergs} \mathrm{s}^{-1}$ for the radio source. Comparison with other X-ray detected radio galaxies shows that the non-thermal component is similar to the nucleus in 3C 98 as opposed to the lobes, which are a factor of 100 weaker (Isobe et al. 2005). The radio galaxy PKS 1343-601 (Tashiro et al. 1998) has a factor of 10 lower non-thermal luminosity from its lobes. Other reported lobe detections include Fornax A with ASCA at $2.8 \times 10^{41} \mathrm{ergs} \mathrm{s}^{-1}$ (Kaneda et al. 1995) and with ROSAT at $2.4 \times 10^{41}$ ergs $\mathrm{s}^{-1}$ (Feigelson et al. 1995). From these comparisons, the non-thermal component in Abell 3120 appears to be higher than is typical of lobes and is therefore likely to be associated with core emission from the radio galaxy. While the spectral index is not well constrained at the low end, the upperlimit with $90 \%$ confidence is 1.56 , also consistent with core emission.

The SUMMS survey (Mauche et al. 2003) at $843 \mathrm{MHz}$ shows a radio source at the position of the Abell 3120 cluster. The source is located at $032156.27+/-1.6-5119$ $35.1+/-1.8$ with flux 47.6+/-1.6 mJy. Figure 7 shows the radio contours overlaid on the $\mathrm{X}$-ray and on the $\mathrm{K}$ band images. The radio source is coincident with the X-ray and K-band emission centers. The resolution of the radio image is $45 \times 45$ arcsec so that the fitted major and minor axes quoted in the catalog, 57.1 and 48.2 arcsec, indicate it may be sightly extended. In fact, the radio image appears to extend nearly 2 arcmin in its longest dimension. The radio contours show a jet-like feature pointing to the N. The X-ray contours in the core (Figure 8) also show a "jet-like" structure pointing to the N, aligned with the radio source morhpology. Both are not aligned with the stellar distribution, which points SE-NW.

Two spatially resolved regions were analyzed to test if there is non-thermal emission: a central region $(\mathrm{r}<15$ arc sec or $20 \mathrm{kpc})$ and an outer annulus, 15 arc sec $<\mathrm{r}<100 \operatorname{arc}$ sec or $126 \mathrm{kpc})$. The background subtracted spectrum for the inner region contained 1003 counts while the spectrum for the outer region contained 3,449 source counts. Both spectra 
were adequate to distinguish between thermal and a non-thermal emission. The results are shown in Table 4. In both regions, a thermal component model was a significantly better fit than a non-thermal component, however, the statistical preference was strongest in the inner region. Thus, it is unlikely that non-thermal emission is from the core of the galaxy, or within $20 \mathrm{kpc}$, as one would expect from an AGN. This is consistent with the spatial analysis in Section 3.1 that showed the harder emission to have a flatter distribution and not centrally peaked, as would be expected from a central AGN.

\subsection{Multiple Redshift Components}

We fit two thermal models that involved multiple redshift components: one with a single thermal and the other with two thermal components. Both provide an improvement compared to the same model with a single redshift. Both multiple redshift models give one component with a redshift comparable to that of Abell 3120 while the second is higher, > 0.12. The model for two thermal components each with its own redshift has an F parameter of 2.7 and significance of $90 \%$. Given the large number of galaxies in this region, the higher redshift component may be from a second cluster along the line of sight.

\section{Summary}

The X-ray image of the Abell 3120 galaxy cluster obtained with Chandra shows emission centered on the central early-type galaxy but extending well beyond, to $158 \mathrm{kpc}$. The X-ray emission appears to be better aligned with the radio morphology on small scales, suggesting that there may be a non-thermal component of X-ray emission. Dividing the radio profile into a hard and soft band shows that a point source is not visible in either band, however, the hard emission has a flatter distribution. A spectral analysis shows 
that the emission from within $20 \mathrm{kpc}$ is thermal and excludes non-thermal emission as the primary component. Beyond the central galaxy, the X-ray emission is aligned with the SE-NW running axis of the asymmetrical galaxy distribution. The emission weighted temperature is $1.93-2.19 \mathrm{keV}$ and the $0.3-10 \mathrm{keV}$ luminosity is $1.23 \times 10^{43} \mathrm{ergs} \mathrm{s}^{-1}$. Modeling the X-ray spectrum excludes purely non-thermal emission. The best fitting model consists of a thermal component and a second component that may be either thermal or non-thermal, with luminosity $\sim 25 \%$ of the total X-ray luminosity. If the second component is non-thermal, it can not be confined to the center and must have a broad, diffuse spatial morphology, similar to the radio source. Since the central $20 \mathrm{kpc}$ and the region beyond have significantly different temperature, $1.28-1.80$ and $1.99-2.58 \mathrm{keV}$, respectively, in the spatial-spectro analysis, this may favor a thermal interpretation for the second spectral component . Abell 3120 has an average abundance, $0.45-0.82$ Solar, which is slightly higher than is typical of rich clusters. In its X-ray properties, Abell 3120 appears to be a poor cluster with Virgo and MKW 4 as peers. 


\section{REFERENCES}

Abell, Corwin, \& Olowin, 1989, ApJS, 70, 1.

Feigelson, E., 1995, ApJ, 449, L149.

Finoguenov, A. et al., 2006, ApJ, 646, 143.

Fleenor et al., M.C., 2006, AJ, 131, 1280.

Fukazawa et al. 2004, ApJ, 606, L109.

Helsdon, S. F., and Ponman, T. J. 2000, MNRAS, 315, 356.

Isobe et al., 2005, ApJ, 632, 781.

Jeltema, T., Mulchaey, J., Lubin, L., Rosati, P., Bhringer, H., 2006, ApJ, 649, 649.

Kaastra, J., Lieu, R., Tamura, T., Paerels, F., den Herder, J., 2003, å, 397, 445.

Kaneda, H., et al. 1995, ApJ, 453, L13.

Matsumota, H, et al., 1997, ApJ, 482, 133

Matsushita et al., 2002, A\&A, 386, 77.

T. Mauch, T. Murphy, H.J. Buttery, J. Curran, R.W. Hunstead, B. Piestrzynska, J.G. Robertson and E.M. Sadler (2003), MNRAS, 342, 1117-1130.

Maddox, S. J., Sutherland, W. J., Efstathiou, G., Loveday, J., 1990, MNRAS, 243, 692.

Mulchaey, J.S., Davis, D., Mushotzky, R., \& Burstein, D., 2003, ApJS, 145, 39.

Oegerle, W. R., Hill, J. M., 2001, AJ, 122, 2858.

Ortiz-Gil, A., Guzzo, L., Schuecker, P., Bhringer, H., Collins, C., 2004, MNRAS, 348, 325 O. 
O'sullivan et al. , 2003, MNRAS, 346, 525.

Reynolds, et al., 1999, ApJ, 521, 99.

Romer, A. K., et al., 2000, ApJS, 126, 209.

Tashiro, et al., 1998, ApJ, 499, 713.

Vikhlinin, A., Forman, W., \& Jones, C., 1999, ApJ, 525, 47.

Zimer, M., Zabludoff, A., \& Mulchaey, J.S., 2003, ApSS, 285, 1. 


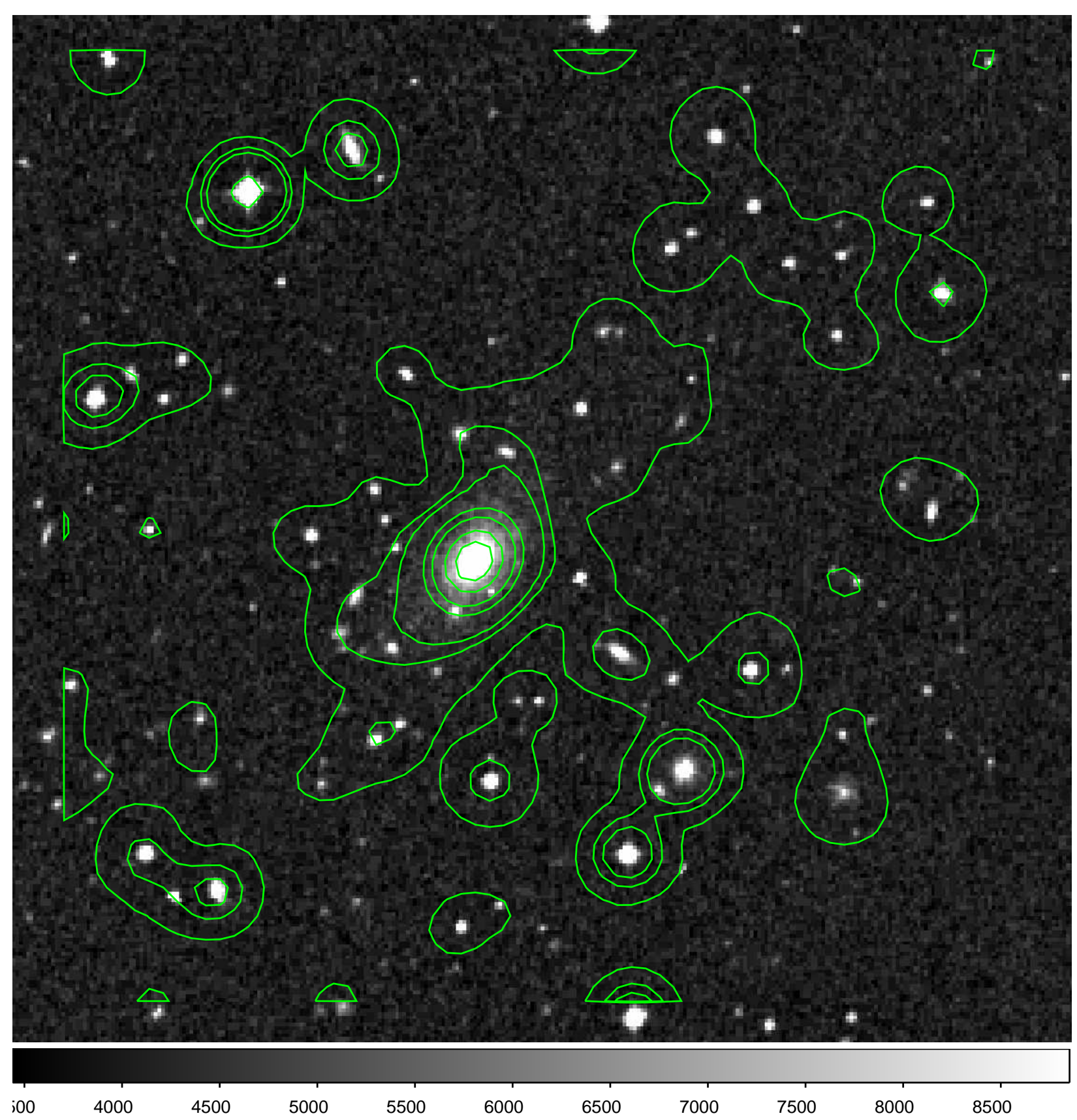

Fig. 1.- Contour map of DSS - R image. An asymmetric galaxy distribution with possible subcondensations is seen, which is typical of a poor, irregular cluster. 
Table 1. A3120 Galaxies With Velocities

\begin{tabular}{cccc}
\hline \hline Galaxy & RA & DEC & Velocity \\
\hline & & & \\
2MASX J03215645-5119357 & $03 \mathrm{~h} 21 \mathrm{~m} 56.4 \mathrm{~s}$ & $-51 \mathrm{~d} 19 \mathrm{~m} 36 \mathrm{~s}$ & 21040 \\
2MASX J03214898-5120197 & $03 \mathrm{~h} 21 \mathrm{~m} 49.0 \mathrm{~s}$ & $-51 \mathrm{~d} 20 \mathrm{~m} 20 \mathrm{~s}$ & 19743 \\
2MASX J03214567-5121156 & $03 \mathrm{~h} 21 \mathrm{~m} 45.7 \mathrm{~s}$ & $-51 \mathrm{~d} 21 \mathrm{~m} 16 \mathrm{~s}$ & 22215 \\
2MASX J03223262-5119548 & $03 \mathrm{~h} 22 \mathrm{~m} 32.6 \mathrm{~s}$ & $-51 \mathrm{~d} 19 \mathrm{~m} 55 \mathrm{~s}$ & 18234 \\
2MASX J03223357-5127128 & $03 \mathrm{~h} 22 \mathrm{~m} 33.6 \mathrm{~s}$ & $-51 \mathrm{~d} 27 \mathrm{~m} 13 \mathrm{~s}$ & 21396 \\
2MASX J03230265-5113333 & $03 \mathrm{~h} 23 \mathrm{~m} 02.6 \mathrm{~s}$ & $-51 \mathrm{~d} 13 \mathrm{~m} 35 \mathrm{~s}$ & 18865 \\
2MASX J03215485-5132207 & $03 \mathrm{~h} 21 \mathrm{~m} 54.8 \mathrm{~s}$ & $-51 \mathrm{~d} 32 \mathrm{~m} 22 \mathrm{~s}$ & 27159 \\
\hline
\end{tabular}

Table 2. Spectral Fits

\begin{tabular}{|c|c|c|c|c|c|c|c|c|}
\hline Model & $\mathrm{n}_{H}\left(\mathrm{~cm}^{-2}\right)$ & $\mathrm{kT}_{1}(\mathrm{keV})$ & Abundance & Redshift $_{1}$ & $\mathrm{kT}_{2}(\mathrm{keV})$ & $\alpha$ & Redshift $_{2}$ & $\chi^{2} /$ dof \\
\hline pow & - & - & - & - & - & - & - & $493.99 / 201$ \\
\hline ray + pow & $0.053-0.111$ & $1.30-1.78$ & $0.19-0.46$ & $0.052-0.082$ & - & $-1.33-1.56$ & - & $220.26 / 200$ \\
\hline ray (two z) & $0.045-0.10$ & $2.05-2.36$ & $0.99-2.41$ & $0.57-0.61$ & - & - & $0.067-0.083$ & $219.29 / 200$ \\
\hline ray + ray (two z) & $0.0017-0.055$ & $2.05-2.85$ & $1.24-2.54$ & $0.053-0.076$ & $1.32-2.42$ & - & $0.140-2.20$ & $214.11 / 199$ \\
\hline
\end{tabular}

Table 3. Spectral Fits - Luminosities

\begin{tabular}{|c|c|c|c|c|c|c|}
\hline Model & Normalization $_{1}$ & Flux $_{1}$ & Luminosity & Normalization $_{2}$ & Flux $_{1}$ & Luminosity \\
\hline ray & $0.00123-0.00139$ & $1.25 \mathrm{e}-12-1.22 \mathrm{e}-12$ & $1.22 \mathrm{e} 43-1.25 \mathrm{e} 43$ & - & - & - \\
\hline ray + pow & $0.00108-0.00185$ & $1.02 \mathrm{e}-12$ & $1.02 \mathrm{e} 43$ & $4.91 \mathrm{e}-7-1.97 \mathrm{e}-5$ & $4.15 \mathrm{e}-13$ & $4.17 \mathrm{e} 42$ \\
\hline ray + ray (one $z$ ) & $0.000191-0.00126$ & $8.84 \mathrm{e}-13$ & $8.87 \mathrm{e} 42$ & $0.000407-0.00107$ & $3.86 \mathrm{e}-13$ & $3.87 \mathrm{e} 42$ \\
\hline ray (two z) & $0.000518-0.00093$ & $8.4 \mathrm{e}-13$ & $8.4 \mathrm{e} 42$ & $0.00045-0.00080$ & $3.7 \mathrm{e}-13$ & $3.7 \mathrm{e} 42$ \\
\hline ray + ray (two z) & $0.00050-0.00081$ & & & $0.00023-0.00049$ & & \\
\hline
\end{tabular}




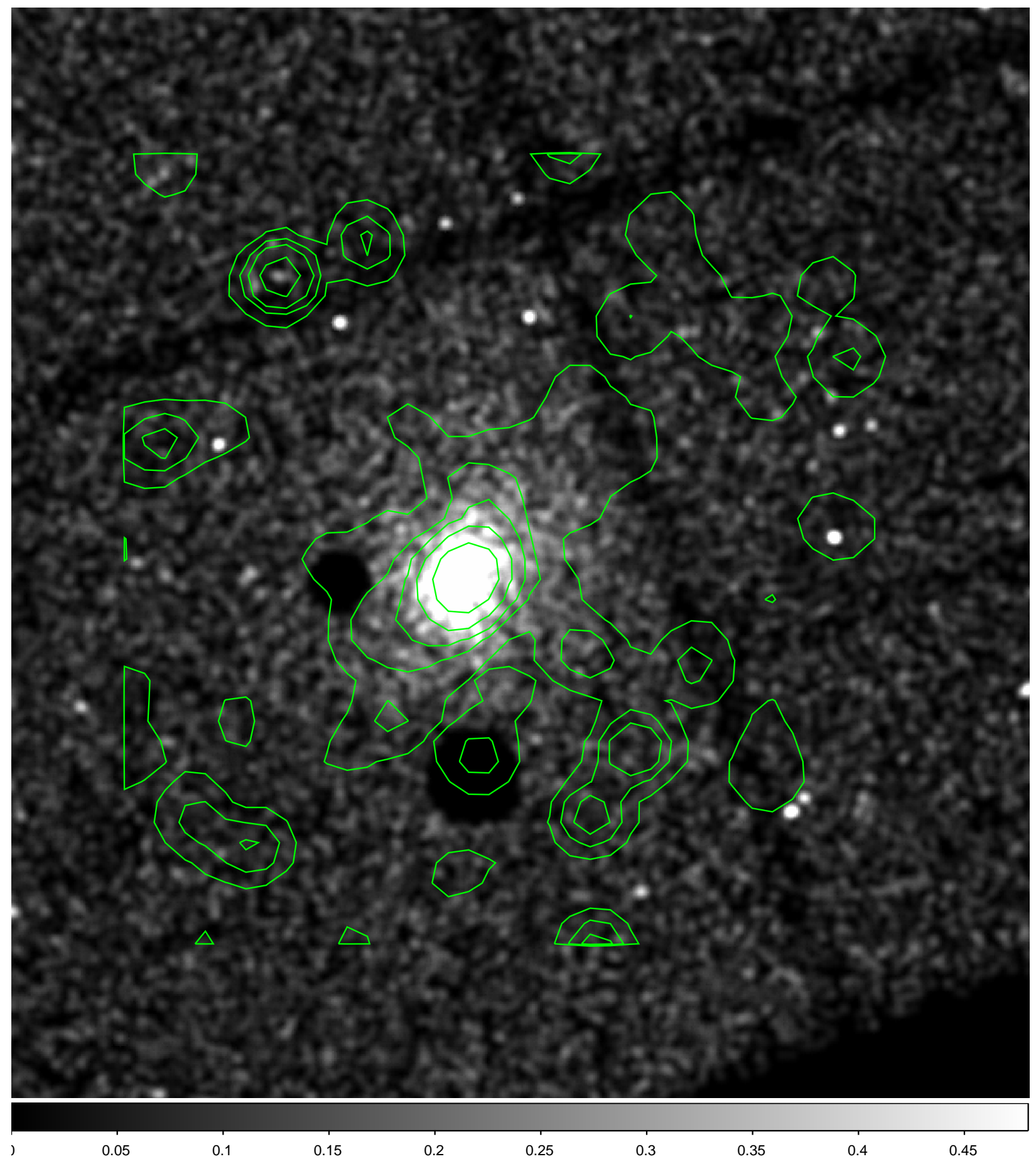

Table 4. Spectral Fits

\begin{tabular}{lccccc}
\hline \hline Region & Model & $\mathrm{n}_{H}\left(\mathrm{~cm}^{-2}\right)$ & $\mathrm{kT}(\mathrm{keV})$ & Abundance & $\chi^{2} / \mathrm{dof}$ \\
\hline \multirow{2}{*}{ Inner } & thermal & $0.026-0.074$ & $1.28-1.80$ & $0.37-0.99$ & $68.78 / 50$ \\
Inner & non-thermal & - & - & - & $105.38 / 51$ \\
Outer & thermal & $0.022-0.085$ & $1.99-2.58$ & $0.35-0.84$ & $220.26 / 200$ \\
Outer & non-thermal & - & - & - & $276.11 / 201$ \\
\hline
\end{tabular}


Fig. 2.- The DSS - $\mathrm{R}$ is shown in contours overlaying the X-ray image. There are 16 catalogued galaxies projected within the X-ray clump.

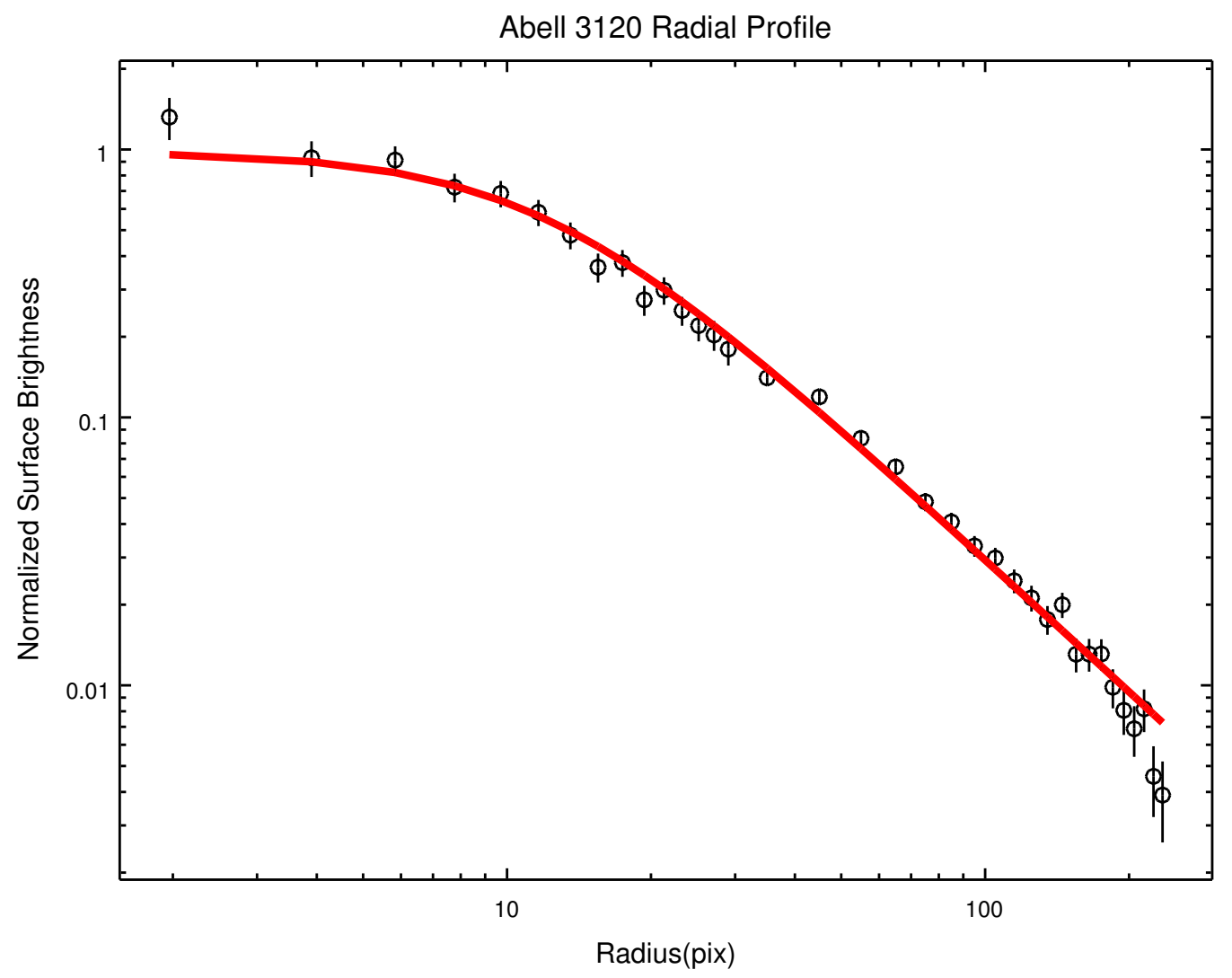

Fig. 3.- The radial profile shows the emission is confined to $158 \mathrm{kpc}$. There may be a slight excess in the center. 


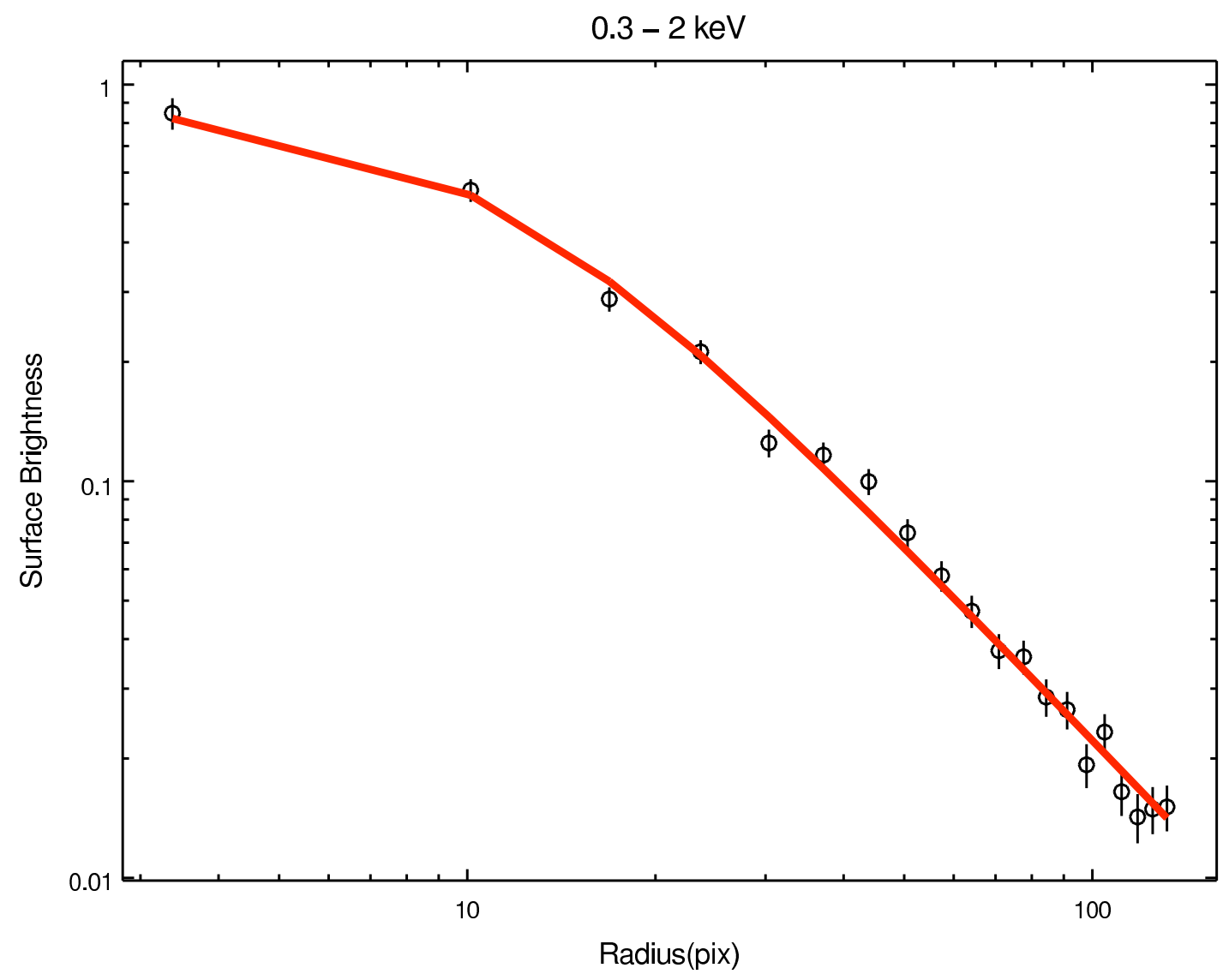

Fig. 4. - The radial profile in the $0.3-2 \mathrm{keV}$ band. 


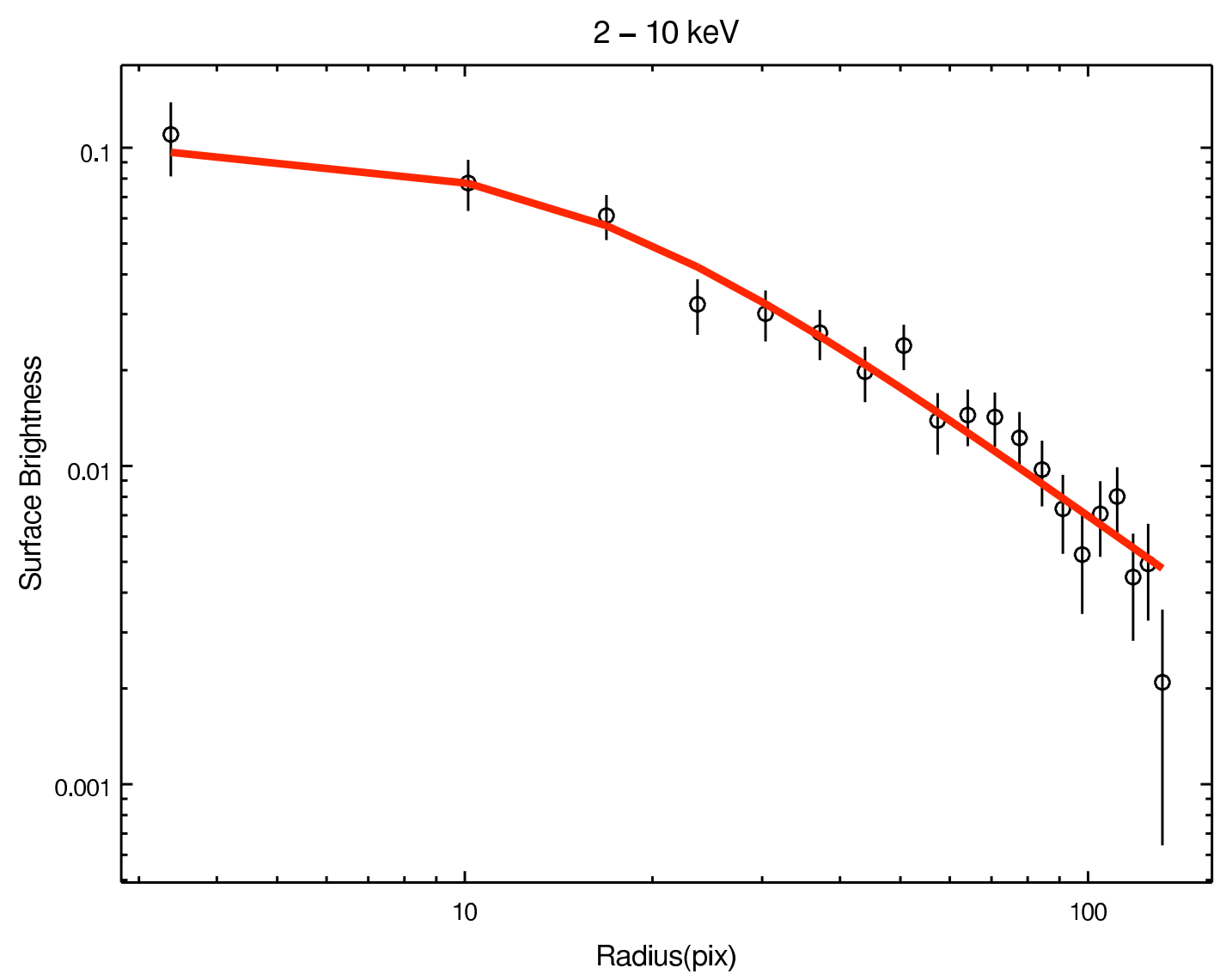

Fig. 5.- The radial profile in the $2-10 \mathrm{keV}$ band appears more extended than the soft band. 


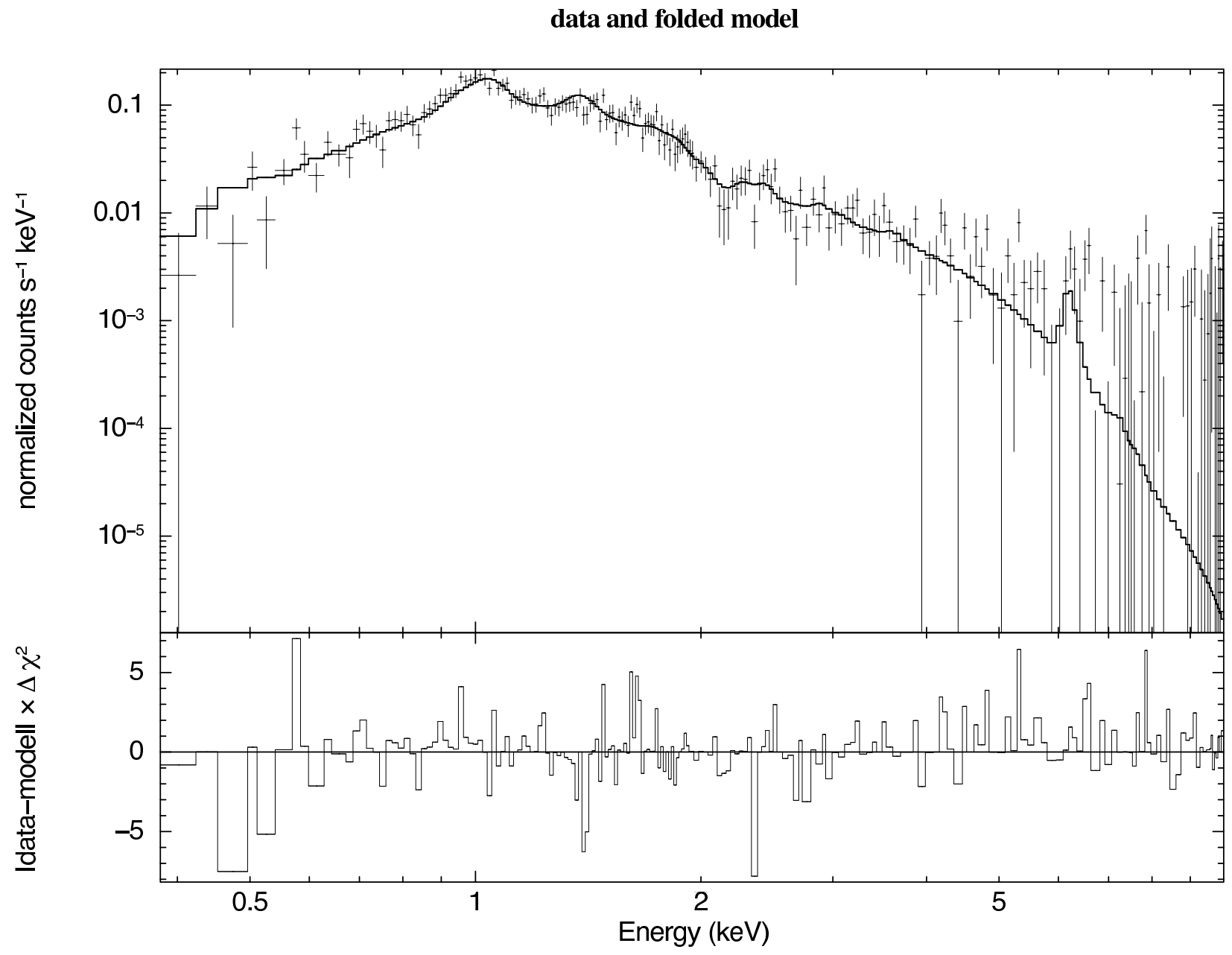

Fig. 6. - Data from $0.3-10 \mathrm{keV}$ is fit by a single thermal, Raymond \& Smith model. Residuals are shown below. 


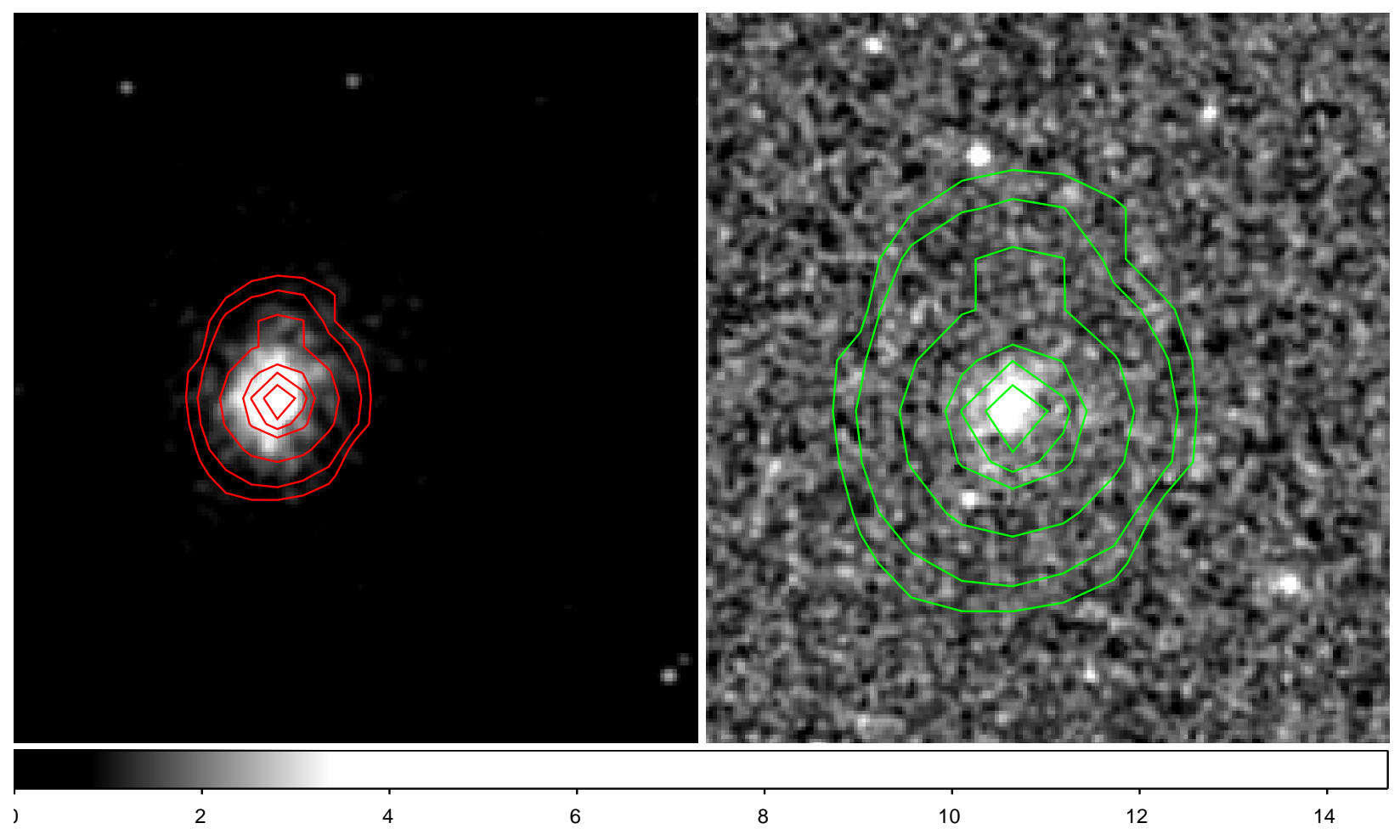

Fig. 7.- Radio contours are overlaid on the X-ray and K band images. 


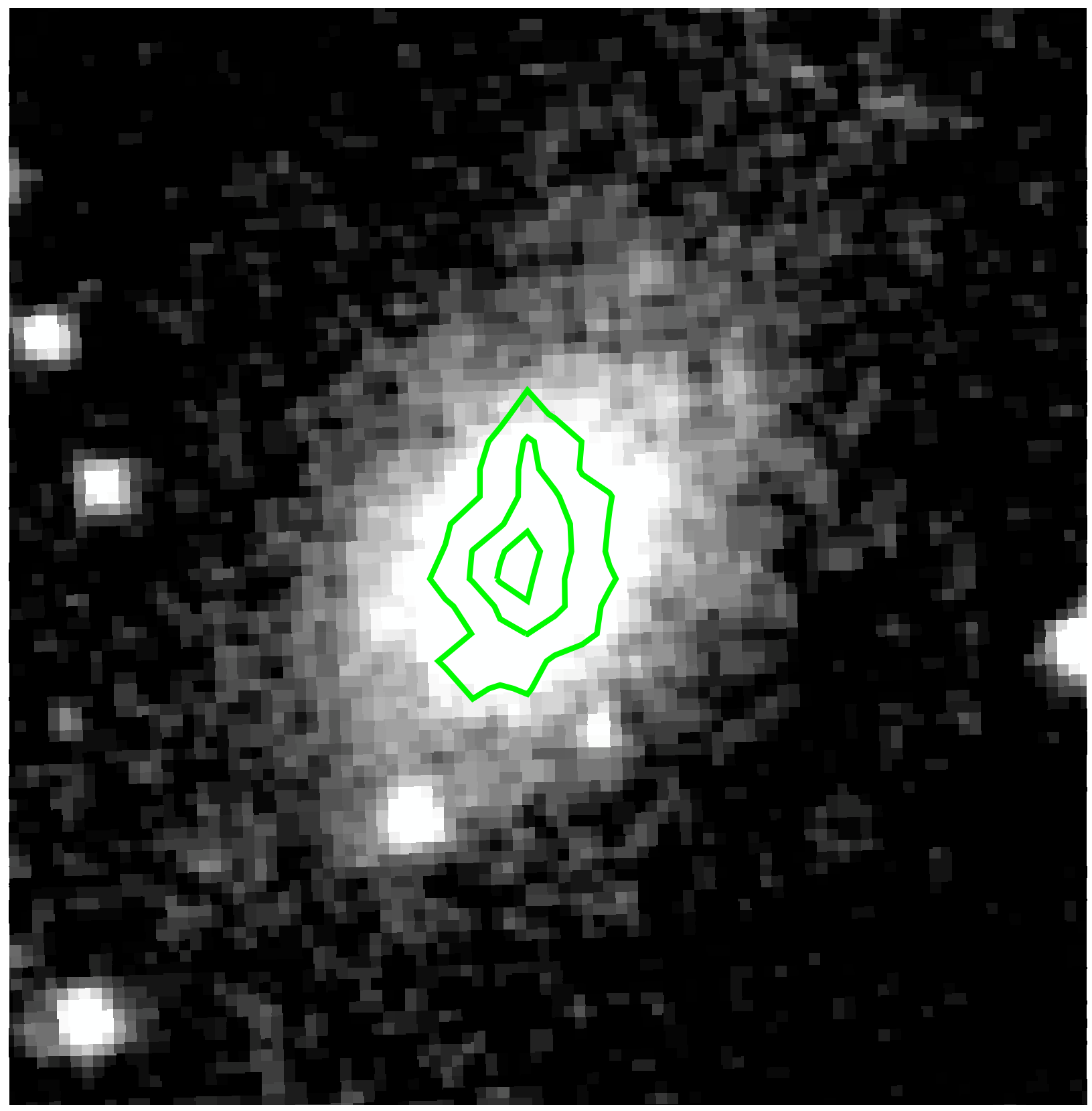

Fig. 8. - X-ray contours are overlayed on the optical image showning the core of the galaxy. A misalignment of the X-ray contours with the stellar light distribution indicates a possible jet to the North. 